\title{
Beware the pitfalls of beauty: High-quality myocardial images with resolution recovery
}

\author{
Kenichi Nakajima, MD, PhD, ${ }^{\text {a }}$ Hiroto Yoneyama, RT, ${ }^{b}$ and Piotr Slomka, $\mathrm{PhD}^{\mathrm{c}}$ \\ a Department of Functional Imaging and Artificial Intelligence, Kanazawa University, Kanazawa, \\ Japan \\ b Department of Radiology Technology, Kanazawa University Hospital, Kanazawa, Japan \\ c AIM Program/Department of Imaging, Cedars-Sinai Medical Center, Los Angeles
}

Received Mar 30, 2019; accepted Apr 1, 2019

doi: $10.1007 / \mathrm{s} 12350-019-01715-\mathrm{x}$

\section{See related article, pp. 236-244}

Myocardial single-photon emission computed tomography (SPECT) is generally recognized as a lowresolution imaging modality, and given that perfusion is a primary assessment by nuclear cardiology, imperfect SPECT images might be acceptable in the era of multimodal cardiac imaging. While we considered the quality of such nuclear images with these factors in mind, we were initially impressed by myocardial perfusion images created using cadmium-zinc telluride (CZT) cameras such as D-SPECT ${ }^{1}$ because the image quality was visually comparable to that generated by positron emission computed tomography (PET). Conventional SPECT images acquired using rotating Anger cameras have an average resolution of $15 \mathrm{~mm}$ under clinical conditions, ${ }^{2}$ and thus the quality is inferior to that highresolution images acquired using magnetic resonance imaging and X-ray computed tomography. The quality of images has become more refined when obtained with CZT cameras with dedicated collimators and reconstruction methods, and thin myocardial walls can be clearly visualized. In addition, new cameras are suitable for obese patients, the dose of injected tracers is lower, images can be rapidly acquired, and dynamic acquisitions are possible in routine clinical investigations. ${ }^{3-6}$

\footnotetext{
Reprint requests: Kenichi Nakajima, MD, PhD, Department of Functional Imaging and Artificial Intelligence, Kanazawa University, Kanazawa 920-8640, Japan; nakajima@med.kanazawa-u.ac.jp J Nucl Cardiol 2021;28:245-8.

$1071-3581 / \$ 34.00$

Copyright (C) 2019 American Society of Nuclear Cardiology.
}

Under most clinical conditions, data obtained with the CZT cameras with dedicated collimators and proprietary iterative reconstruction, which includes resolution recovery for the collimator-related loss in spatial resolution and also can include the cardiac anatomic model (heart prior), which constrains the shape and thickness of left ventricular walls ${ }^{3}$ for Spectrum Dynamics (SD) (Cesarea, Israel) cameras, seems to work well, providing high-resolution data acquisition and processing. Since myocardial thickness ranges from a few to $10 \mathrm{~mm}$ on echocardiograms, a method of reconstructing thin walls using appropriate models is desirable in nuclear imaging. Even with gated SPECT imaging, while cardiac and respiratory motion is an important issue that remains to be resolved, higher resolution is preferable in the era of multi-modal cardiac imaging.

Nevertheless, we have found that the advanced SD reconstruction of D-SPECT images incorporating resolution recovery and heart-priors assumptions may not always work optimally in certain clinical cases. Figure 1 shows a 39-year-old female patient with chest symptoms, and initial SPECT images were created with SD reconstruction. Thin walls are obvious with ${ }^{99 \mathrm{~m}} \mathrm{Tc}$ tetrofosmin, and hypertrophy was absent at first glance. However, the patient had suspected hypertrophic cardiomyopathy. Therefore, we reconstructed the images using ordered-subset expectation-maximization (OSEM) without resolution recovery or anatomical assumptions and clearly identified a thick anteroseptal wall. This patient also underwent simultaneous fatty acid imaging with ${ }^{123}$ I-beta-methyl-idiophenylpentadecanoic acid (BMIPP) and perfusion imaging to identify ischemic memory. The difference in anteroseptal wall thickness was obvious, and heterogeneous anteroseptal uptake was also discernible. To visualize a thickened anteroseptal wall when using SD reconstruction, we encountered 


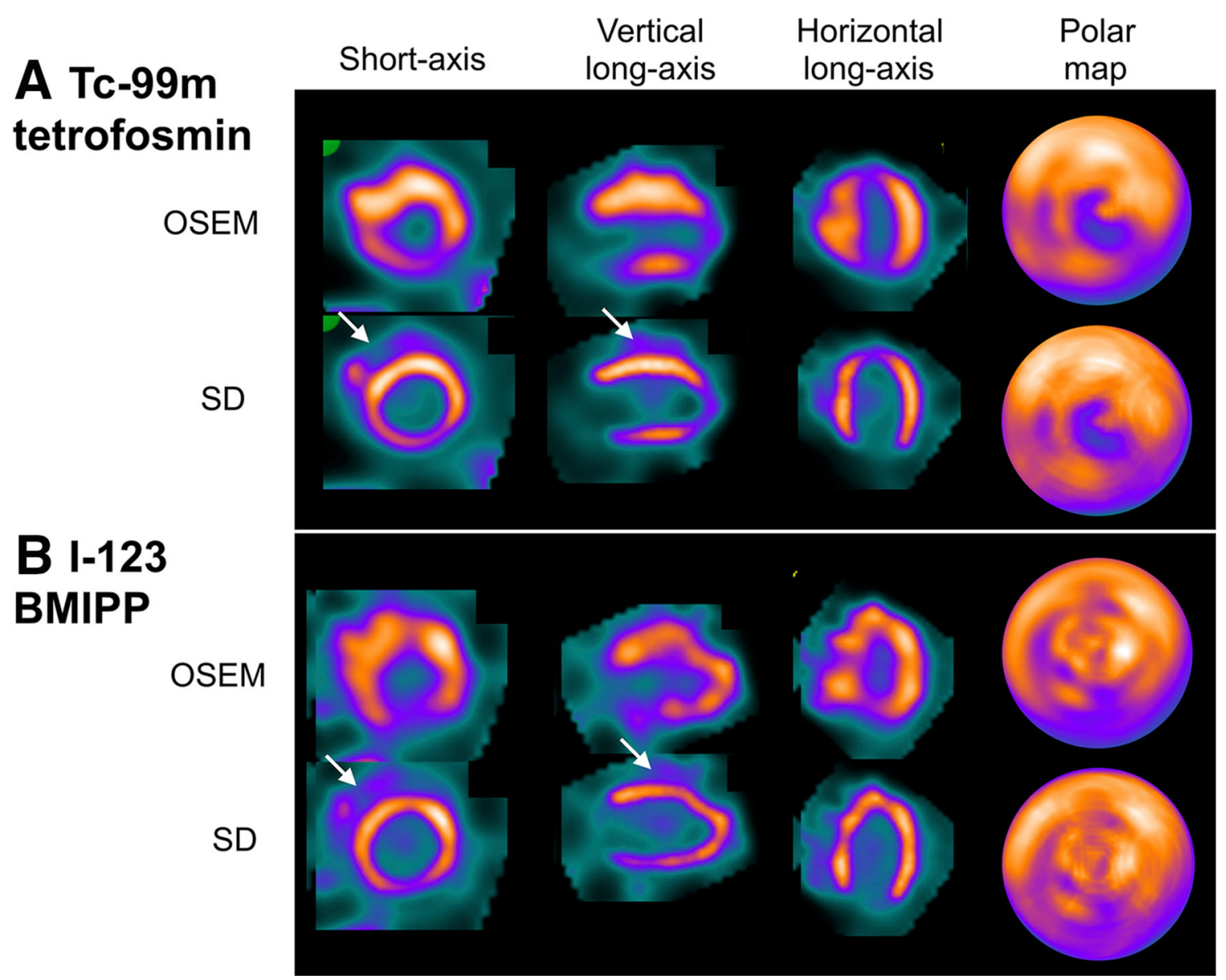

Figure 1. Images of patient with hypertrophic cardiomyopathy. ${ }^{99 \mathrm{~m}} \mathrm{Tc}$-tetrofosmin (A) and ${ }^{123} \mathrm{I}-$ BMIPP (B) images were simultaneously acquired using dual-isotope SPECT. Anteroseptal wall thickness differs between OSEM and SD reconstruction. Slight amount of unusual activity (arrows) evident outside anteroseptal walls suggests inappropriate SD reconstruction. Polar maps are similar for both methods.

peri-myocardial activity that was not evident in ordinary reconstructed images.

Such clinical findings are indeed supported by a phantom study by Tsuboi et al. ${ }^{7}$ which is published in this issue of the Journal of Nuclear Cardiology. That study investigated an asymmetric septum and lateral wall ranging in thickness from 10 to $40 \mathrm{~mm}$ using proprietary SD and standard OSEM reconstruction. The calculated left ventricular volume was higher after SD reconstruction, and wall thickness significantly differed between the two types of reconstruction. A clinical analysis using ${ }^{201} \mathrm{Tl}$ and ${ }^{99 \mathrm{~m}} \mathrm{Tc}$ perfusion imaging tracers and OSEM and SD reconstruction found that the average wall thickness measured at FWHM was 28 and $22 \mathrm{~mm}$, respectively, and the calculated left ventricular volumes were 65 and $71 \mathrm{~mL}$, respectively. Although the difference in these volumes was relatively small (for example $83 / 77 \mathrm{~mL}=1.08$ for $\mathrm{SD}$ and OSEM methods, respectively, when a phantom had a septal thickness of $40 \mathrm{~mm}$ ) and the correlation of volumes between SD and OSEM was excellent $(r=0.99)$, they concluded that OSEM was more accurate than SD for patients with hypertrophic cardiomyopathy. ${ }^{7}$ The most important finding in that study was that the appearance of wall thickness differed depending on the reconstruction methods. While the thick walls of the phantom could be reconstructed using both SD and OSEM, they looked quite different, as suggested by a cross-sectional count profile curves across the septum. Since their study does not include anatomical standards, the effect of this reconstruction on the measured wall thickness should be further evaluated in clinical multimodality studies with CT or MRI.

The SD reconstruction was specifically designed for the SD camera geometry with nine arrays of detectors that permit cardiac region-centric acquisition and for the 
aggressive resolution recovery for the resolution lost by the high-sensitivity collimator. Iterative reconstruction permits the restoration of resolution by including a model of the collimator response characteristics and geometry within the system model. ${ }^{8}$ The manufacturer reports that the spatial resolution averages $4.36 \mathrm{~mm}$ at FWHM when resolution recovery is model-based -assuming certain anatomical contraints. ${ }^{9}$ For standard static and gated myocardial perfusion imaging using SD reconstruction, the cardiac filter, iterations, inter-iterative, post- and temporal filters are all adjusted for the clinical use by the vendor. During such processing, the weight of the cardiac filter seems to adjust according to the shape of the myocardial walls. The vendor also provides an SD-less filter without low-pass filtering for high-count studies and other reconstruction options for small hearts. In contrast, the OSEM algorithm provides standard reconstructed images for clinical studies without such advanced optimizations.

Whereas SPECT images with resolution recovery and anatomical constraints might work well in most clinical situations, caution might be advised when reconstructing images using the D-SPECT algorithm in the face of atypical myocardial shapes and count distributions. For example, asymmetric wall thickening is characteristically evident in the septum of patients with hypertrophic cardiomyopathy, and some patients might have apical hypertrophy. When myocardial hypertrophy is not anticipated before a nuclear study, a false impression might arise that could lead to misdiagnosis, when advanced reconstruction options are used. A large papillary muscle might also be obscured by resolution recovery, although this has not yet been systematically confirmed. When a myocardial defect is large, for example in ${ }^{123}$ I-BMIPP myocardial fatty acid images of an acute ischemic episode, the adequacy of wall shape also requires confirmation.

One challenge, for example, is imaging large defects often seen in ${ }^{123}$ I-meta-iodobenzylguanidine

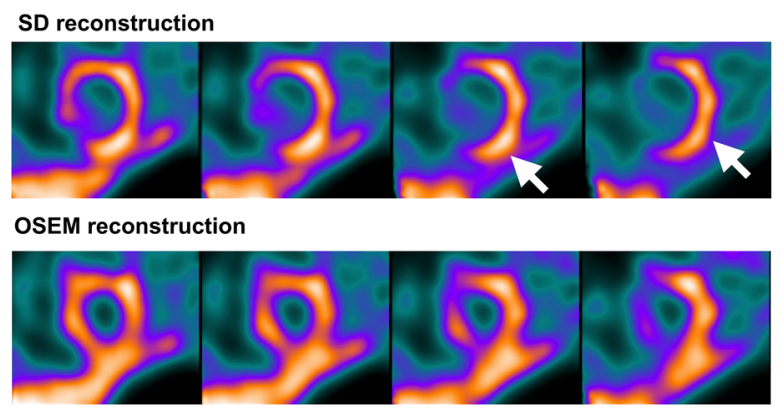

Figure 2. Late phase ${ }^{123} \mathrm{I}-\mathrm{mIBG}$ images acquired at three hours after injection. Activity on diaphragm is visualized as part of inferior wall after SD reconstruction, whereas inferior wall is separately visualized after OSEM reconstruction.
( $m$ IBG) images as shown in Figure 2. Since most of the inferior wall is defective, heart-priors assumption or modeling the myocardial wall might create artificial myocardial contours incorporating diaphragmatic activity. Both ${ }^{123}$ I-BMIPP and ${ }^{123}$ I- $m$ IBG have been utilized with conventional cameras in hypertrophic cardiomyopathy since 1990s in Japan and showed regional abnormality in hypertrophic regions. ${ }^{10,11}$ Further studies of this tracer will continue with new high-resolution SPECT in future. For example, the accuracy of hot imaging in acute myocardial infarction such as ${ }^{99 \mathrm{~m}} \mathrm{Tc}$ pyrophosphate with these new cameras remains to be determined.

We recognize that resolution recovery as used in SD reconstruction plays an important role in perfusion imaging. Qutub et al. ${ }^{12}$ reported on a different camera system that the resolution recovery was beneficial in increasing concordance between PET and SPECT. The agreement with ${ }^{82} \mathrm{Rb}$ PET for iterative reconstruction with resolution recovery was significantly better with than that without resolution recovery, and the clinical diagnosis was also improved by resolution recovery. But these advanced options may nevertheless be a cause of confusion in hypertrophic cardiomyopathy as shown by Tsuboi et al. Thus, there is a clinical dilemma; advanced reconstruction with resolution recovery and anatomical priors is beneficial for the diagnosis of coronary artery disease, but standard methods may need to be used for the evaluation of less common patterns such as hypertrophy.

The effects of resolution recovery on the accuracy of left ventricular volume and ejection fraction can also be evaluated in patients with hypertrophy and large defects. The evaluation of the myocardial volumes depends not only on the reconstruction methods but also on the edge detection approaches of different software algorithms. ${ }^{13-15}$ Myocardial shape and wall can be determined using, for example, an ellipsoid, or activeshape model of the ventricles. ${ }^{16-18}$ Symmetric wall thickness can be assumed by some quantitation software during processing. This sort of left ventricular modeling contributes to the reproducible detection of the edge even of bizarre shapes and defects in the myocardium. On the other hand, contour detection based on a threshold method might work for asymmetric walls, but detecting the wall is difficult in segments with large perfusion defects. When accurate measurement is required for $\mathrm{LV}$ volumes and the myocardial mass, the software should be carefully applied considering these factors. Since quantitation software for functional analysis is now being applied to patients with various pathologies using CZT camera, ${ }^{19,20}$ careful quality control of the methodology and further validation studies will become more important. 
In general, as the quality of images reconstructed using the resolution recovery and heart-priors assumption is superb due to its high-resolution reconstruction algorithm and highly tuned approach, many nuclear physicians and cardiologists prefer such high-resolution high-quality images for daily clinical practice. However, not all routine imaging tasks require such exceedingly high resolution generated by these algorithms and it may be safer to utilize more standard methods in some clinical scenarios. In particular, secondary reconstruction by OSEM is recommended for D-SPECT when asymmetric hypertrophy and large defects are suspected.

\section{Acknowledgements}

Data for Figure 1 were obtained from Matto Ishikawa Hospital, Hakusan Japan (Courtesy of Dr. Kunihiko Yokoyama) and data for Figure 2 were from Kanazawa University Hospital. The authors thank Mr. Yasuhiro Suzuki, Sakakibara Memorial Hospital for providing constructive comments. We also appreciate Norma Foster for editorial assistance.

\section{Disclosure}

None to disclose.

\section{References}

1. Berman DS, Kang X, Tamarappoo B, Wolak A, Hayes SW, Nakazato R, et al. Stress thallium-201/rest technetium- $99 \mathrm{~m}$ sequential dual isotope high-speed myocardial perfusion imaging. JACC Cardiovasc Imaging. 2009;2:273-82.

2. Imbert L, Poussier S, Franken PR, Songy B, Verger A, Morel O, et al. Compared performance of high-sensitivity cameras dedicated to myocardial perfusion SPECT: A comprehensive analysis of phantom and human images. J Nucl Med. 2012;53:1897-903.

3. Slomka PJ, Patton JA, Berman DS, Germano G. Advances in technical aspects of myocardial perfusion SPECT imaging. J Nucl Cardiol. 2009;16:255-76.

4. Ben-Haim S, Kennedy J, Keidar Z. Novel cadmium zinc telluride devices for myocardial perfusion imaging-technological aspects and clinical applications. Semin Nucl Med. 2016;46:273-85.

5. Reiber JHC, De Sutter J, Schoenhagen P, Stillman AE, Vande Veire NRL. Cardiovascular imaging 2016 in the International Journal of Cardiovascular Imaging. Int J Cardiovasc Imaging. 2017;33:761-70.

6. Claudin M, Imbert L, Djaballah W, Veran N, Poussier S, Roch V, et al. Routine evaluation of left ventricular function using CZTSPECT, with low injected activities and limited recording times. J Nucl Cardiol. 2018;25:249-56.
7. Tsuboi K, ANagai A, Shibutani T, Onoguchi M. Optimal choice of OSEM and SD reconstruction algorithms in CZT SPECT for hypertrophic cardiomyopathy patients. J Nucl Cardiol 2019. http s://doi.org/10.1007/s12350-019-01677-0 (Epub ahead of print).

8. Erlandsson K, Kacperski K, van Gramberg D, Hutton BF. Performance evaluation of D-SPECT: A novel SPECT system for nuclear cardiology. Phys Med Biol. 2009;54:2635-49.

9. Patton JA, Slomka PJ, Germano G, Berman DS. Recent technologic advances in nuclear cardiology. J Nucl Cardiol. 2007;14:501-13.

10. Nakajima K, Bunko H, Taki J, Shimizu M, Muramori A, Hisada K. Quantitative analysis of 123I-meta-iodobenzylguanidine (MIBG) uptake in hypertrophic cardiomyopathy. Am Heart J. 1990;119:1329-37.

11. Ohtsuki K, Sugihara H, Kuribayashi T, Nakagawa M. Impairment of BMIPP accumulation at junction of ventricular septum and left and right ventricular free walls in hypertrophic cardiomyopathy. $\mathrm{J}$ Nucl Med. 1999;40:2007-13.

12. Qutub MA, Dowsley T, Ali I, Wells RG, Chen L, Ruddy TD, et al. Incremental diagnostic benefit of resolution recovery software in patients with equivocal myocardial perfusion single-photon emission computed tomography (SPECT). J Nucl Cardiol. 2013;20:545-52.

13. Germano G, Kavanagh PB, Slomka PJ, Van Kriekinge SD, Pollard G, Berman DS. Quantitation in gated perfusion SPECT imaging: The Cedars-Sinai approach. J Nucl Cardiol. 2007;14:433-54.

14. Garcia EV, Faber TL, Cooke CD, Folks RD, Chen J, Santana C. The increasing role of quantification in clinical nuclear cardiology: The Emory approach. J Nucl Cardiol. 2007;14:420-32.

15. Ficaro EP, Lee BC, Kritzman JN, Corbett JR. Corridor4DM: The Michigan method for quantitative nuclear cardiology. J Nucl Cardiol. 2007;14:455-65.

16. Germano G, Kavanagh PB, Su HT, Mazzanti M, Kiat H, Hachamovitch R, et al. Automatic reorientation of three-dimensional, transaxial myocardial perfusion SPECT images. J Nucl Med. 1995;36:1107-14.

17. Faber TL, Cooke CD, Folks RD, Vansant JP, Nichols KJ, DePuey $\mathrm{EG}$, et al. Left ventricular function and perfusion from gated SPECT perfusion images: An integrated method. J Nucl Med. 1999;40:650-9.

18. Lomsky M, Richter J, Johansson L, El-Ali H, Astrom K, Ljungberg $\mathrm{M}$, et al. A new automated method for analysis of gatedSPECT images based on a three-dimensional heart shaped model. Clin Physiol Funct Imaging. 2005;25:234-40.

19. Tsai SY, Wang SY, Shiau YC, Wu YW. Mechanical dyssynchrony and diastolic dysfunction are common in LVH: A pilot correlation study using Doppler echocardiography and CZT gated-SPECT MPI. Sci Rep. 2018;8:4182.

20. Gimelli A, Liga R, Menichetti F, Soldati E, Bongiorni MG, Marzullo P. Interactions between myocardial sympathetic denervation and left ventricular mechanical dyssynchrony: A CZT analysis. J Nucl Cardiol 2017;26(2):509-518. https://doi.org/10.1 007/s12350-017-1036-3 (Epub ahead of print).

Publisher's Note Springer Nature remains neutral with regard to jurisdictional claims in published maps and institutional affiliations. 